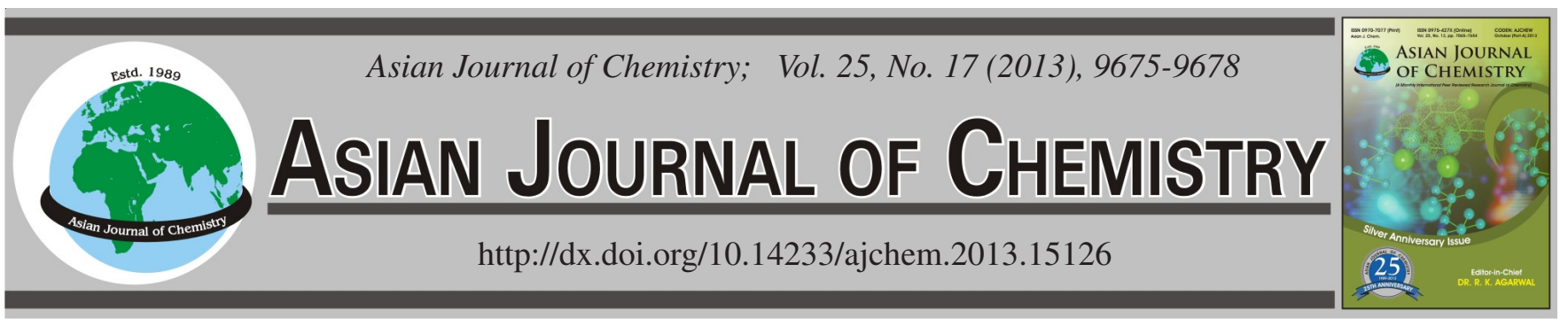

\title{
Lipase-Catalyzed Synthesis of Ethyl Hexanoate in Microemulsion System
}

Zhongein Tan, XiaOXiang Han ${ }^{*}$, XiaOli Hu, Huan Du and XiuXiu Bao

Department of Applied Chemistry, College of Food Science \& Biotechnology Engineering, Zhejiang Gongshang University, Hangzhou 310035, P.R. China

*Corresponding author: Tel: +86 571 88071024-7581; E-mail: hxx74@126.com; han-xx74@163.com

(Received: 10 January 2013;

Accepted: 11 October 2013)

AJC-14252

This paper studied lipase-catalyzed synthesis of ethyl hexanoate in dodecylbenzenesulfonic acid/isooctane/water microemulsion system. The effect of several parameters, such as $\mathrm{w}_{0}\left(\left[\mathrm{H}_{2} \mathrm{O}\right] /[\right.$ surfactant $\left.]\right)$ value, reaction time, reaction temperature, oil phase solvent, buffer solution $\mathrm{pH}$ value of microemulsion system on the esterification have been investigated. The results showed that the best experimental conditions for catalytic synthesis ethyl hexanoate were as follows: $\mathrm{w}_{0}=4$, reaction time $4 \mathrm{~h}$, reaction temperature $40^{\circ} \mathrm{C}$, buffer solution $\mathrm{pH}$ 7. Under these conditions, the conversion of ethyl hexanoate can reach $98.5 \%$. Lipase-catalyzed synthesis of ethyl hexanoate in dodecylbenzenesulfonic acid inverse microemulsion system has triple mechanism, namely acid catalyzes, microemulsion catalyzes and enzyme catalyzes.

Key Words: Esterification, Dodecylbenzenesulfonic acid, Microemulsion, Lipase.

\section{INTRODUCTION}

The method of producing ester catalyzed by lipase, in the quality and the smell, is better than traditional method of synthesizing products which have natural quality ${ }^{1-3}$. In the future, the application of lipase is an important way to catalytically synthesize ester. Ethyl hexanoate is colourless or transparently light yellow liquid and has strong fruit and wine aroma, widely used in food, beverage, tobacco and wine industry and also used for cosmetics, soap, shampoo, as everyday life product spices raw material. Ethyl hexanoate not only has a broad range of applications, but also with the development of the related industries, especially the increasing production of wine renders the increasing demands of ethyl hexanoate. The output of ethyl hexanoate only used in the luzhou-flavor liquor reached 3000 tons per year all over the country and the production value achieved hundreds of millions ${ }^{4}$. At present, ethyl hexanoate is mainly prepared by hexanoic acid and ethanol using sulfuric acid as catalyst in industry. But this method has many side effects, such as serious equipment corrosion, complex acid liquid post-treatment, difficult catalyst regeneration and doesn't belong to the green process. Using these catalysts such as solid acid, metal salt, alkaline lipase ${ }^{5-7}$ could solve traditional catalysts' shortcomings to some extent, but there were still some defects such as the low relative activity, easily accumulating carbon on the surface, low acid density and unequal acid intensity distribution, which limited their applications.
The thermodynamically stable, isotropic, optically transparent, highly decentralized microemulsion system that consists of surfactant, surface active agent, oil and water in certain proportion, as a micro reactor, has been widely used in many fields ${ }^{8-14}$. Not only it can expand the reaction contact area to improve the reaction rate and the conversion, but also the water droplets of inverse microemulsion system can simulate the natural environment of enzyme, make the enzyme maintains catalytic activity and stability in the water environment. Therefore, inverse microemulsion system is suitable for lipase catalytic reaction. At present, lipase catalyzing synthesis of short chain fatty acid ester in microemulsion has also been reported in the literature ${ }^{15-17}$. In this paper, we prepared the dodecylbenzenesulfonic acid (DBSA)/isooctane/water microemulsion system and investigated several single factors in this system on the esterification caproic acid and ethanol. The results showed that lipase-catalyzed synthesis of ethyl hexanoate in DBSA inverse microemulsion system with acid catalysis, microemulsion catalysis and enzyme catalysis triple mechanism.

\section{EXPERIMENTAL}

Dodecylbenzenesulfonic acid (DBSA, $\approx 97 \%$ purity) was purchased from Tokyo Kasei Kogyo Co. Ltd. (Japan). Candida rugosa lipase (Type VII) was obtained from Sigma Co. Ltd. Isooctane, cyclohexane, hexane, caproic acid, butyric acid, isoamyl acid, ethanol, $n$-butanol and isoamyl alcohol were all 
from Shanghai chemical reagent factory (China). All the other chemicals used were analytical grade and were used without further purification.

Preparation of lipase microemulsion and the biggest reaction point: DBSA surfactants acts as a component, oil phase acts as the second component. First, make the mixture of surfactants and oil, the oil content in this mixture is called starting oil content. Then use a liquid gun to titrate dissolved lipase in water to the mixture in the magnetic blender, to dissolve all surfactants at the first phase change point, where solution of this system is homogeneous, generating microemulsion and presenting clear transparent state. After that, continue adding water, sample transforming from transparent to turbid, this time at the second phase change point, among a clear solution called microemulsion.

Change with the starting oil content of the surfactant oil mixture and repeat the above titration procedure, get different starting oil content samples at the phase change point. Then calculate the mass ratio of three components surfactant, oil, water at phase change point, by using Origin software to sketch three-phase figure and determine the formation regional of microemulsion system ${ }^{18}$.

Take several special points at microemulsion area, work out every component mass according to the mass ratio, prepare the microemulsion system. In the same reaction time and under the same temperature conditions, use lipase catalyzed synthesis of ethyl hexanoate in this system, respectively. According to the conversion, get the biggest reaction point of this microemulsion area, this point aims to explore the single factor influence point of ethyl hexanoate synthesis reaction.

Lipase-catalyzed synthesis of ethyl hexanoate in microemulsion system: Add microemulsion system formed by three components into $50 \mathrm{~mL}$ round bottom flask, stir continuously in the magnetic blender for $0.5 \mathrm{~h}$ to form a clear, transparent microemulsion system. Then esterification was carried out in the above microemulsion system by addition of $3 \mathrm{mmol}$ caproic acid and $6 \mathrm{mmol}$ ethanol. After some time, samples were withdrawn from the reaction medium and analyzed subsequently by GC.

Gas chromatography analysis: After being analyzed by gas chromatography, take $200 \mu \mathrm{L}$ reaction mixtures and a certain amount of butyl acetate as internal standard into a test tube, to be dissolved with $5 \mathrm{~mL}$ ethanol, attaining a processed samples. Qualitative study by retention time and quantitative study by internal standard method, calculate the conversion per cent of the hexanoic acid.

Gas chromatograph was equipped with a FID detector using an Agilent technologies HP-5 column $(30 \mathrm{~m} \times 0.32 \mathrm{~mm}$ $\times 0.25 \mathrm{~mm}$ ). Injection port temperature is $240^{\circ} \mathrm{C}$, detector temperature is $260^{\circ} \mathrm{C}$, carrier gas is nitrogen. The temperature program used began at $50^{\circ} \mathrm{C}$ for $2 \mathrm{~min}$, then increased to $260^{\circ} \mathrm{C}$ at a gradient of $20^{\circ} \mathrm{C} / \mathrm{min}$, maintained for $20 \mathrm{~min}$.

\section{RESULTS AND DISCUSSION}

Effects of microemulsion $\mathbf{w}_{\mathbf{0}}$ value: The three-phase diagram of DBSA/isooctane/water was shown in Fig. 1. Fig. 1 showed that the microemulsion area was small. We also found that DBSA and isooctane could form transparent solution

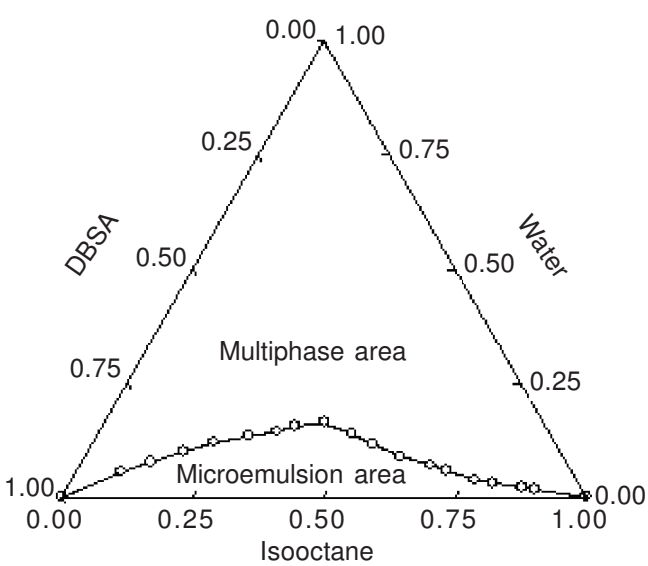

Fig. 1. Three-component phase diagram of DBSA/isooctane/water microemulsion system

without drop water in any ratio, it didn't have the first phase transition point. All experiment were studied in this microemulsion area.

The water content in microemulsion system expressed by $\mathrm{w}_{0}$, defined as the amount of water divided the amount of DBSA and had an important influence to esterification. We have studied the effect of $w_{0}$ on the esterification conversion (Fig. 2). Fig. 2 showed that the conversion of hexanoic acid increased with increasing the amount of water. When $\mathrm{w}_{0}=4$, $98.5 \%$ of hexanoic acid was converted into ethyl hexanoate in $4 \mathrm{~h}$. However, when the $\mathrm{w}_{0}$ exceeded 4 , the conversion of hexanoic acid and yield of ethyl hexanoate decreased slightly.

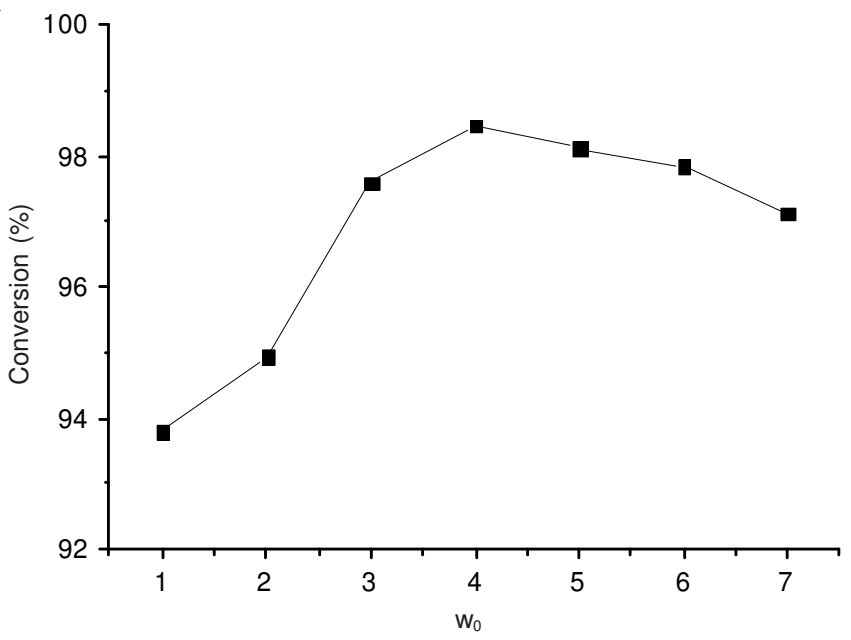

Fig. 2. Results of esterification under different water content $\mathrm{w}_{0}$

The reason of forming this phenomenon as follows: When $\mathrm{w}_{0}$ was low, the water content of microemulsion system was low, almost all water in system was combined with surfactant to form microemulsion and kept enzyme activity. The amount of water was small which inhibited the enzyme activity. The conversion of hexanoic acid was low. With the rising of $\mathrm{w}_{0}$, the water content increased, the extra water increased the activity of enzyme. Also the produced water in reaction system could easily enter the water mocrodomain and enlarge the water droplet. In microemulsion system, the organic reactions occurred at interface. The larger interface area of microemulsion provided more opportunity of enzyme and 
substrate collision frequency and more completely of the reaction. Esterification was a dehydration reaction, when $\mathrm{w}_{0}$ was high, so water content was too much in the reaction system, which made the microemulsion transform into emulsifiable or unstable system and the phase interface area decreased. This would lead to the reaction equilibrium towards reverse opposite direction, which facilitated ester hydrolysis and reduced the conversion of acid.

Effects of reaction temperature: Generally, high temperature could contribute to the enhancement of reaction rate as well as conversion efficiency. Similar results were also observed in our enzyme system. The effect of reaction temperature on the esterification of hexanoic acid with ethanol was shown in Fig. 3. In the systematic condition of $\mathrm{w}_{0}=4$ and molar ratio of acid to ethanol $1: 2,87.5 \%$ yield of ethyl hexanoate was obtained at $30^{\circ} \mathrm{C}$ for $4 \mathrm{~h}$. The yield of ethyl hexanoate increased with a rise in temperature and reached a maximum of $98.5 \%$ at $40{ }^{\circ} \mathrm{C}$. Nevertheless, further increase of temperature resulted in the decrease of yield of ethyl hexanoate. It was owing to every enzyme had its flexible and optimal reaction temperature and more or less temperature would constrain the speed of enzyme catalytic reaction and the stability of enzyme. The enzyme catalytic reaction was faster while the stability of enzyme becomed worse with higher temperature. The optimum temperature was that the enzyme kept high activity and stability.

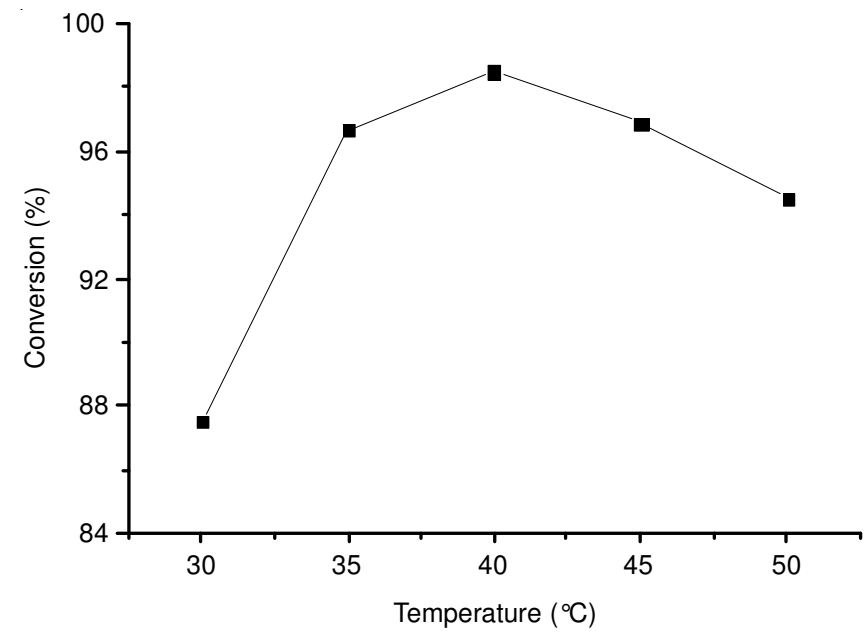

Fig. 3. Results of esterification under different reaction temperature

Effects of buffer solution pH value: There were many side chain groups of alkaline and acid amino acids in lipase molecules, these groups had different solubility in acidic or alkaline solution. The solubility had an important effect on enzyme combine with substrate and the next reaction, could also change the space conformation of the enzyme and enzyme activity. That was, changing the buffer solution $\mathrm{pH}$ value of microemulsion system would alter the conformation of enzymes, which transformed the enzyme catalytic effect. Fig. 4 showed the yield of ethyl hexanoate operating at desired $\mathrm{pH}$ value and $\mathrm{w}_{0}=4$ at $40{ }^{\circ} \mathrm{C}$. It could be found $98.5 \%$ of ethyl hexanoate was achieved at $\mathrm{pH}$ of 7 and the yield of ethyl hexanoate became feeble while the more or less $\mathrm{pH}$ was adjusted. Thus, considering active environment and activity, the optimum buffer solution $\mathrm{pH}$ value should be around 7 .

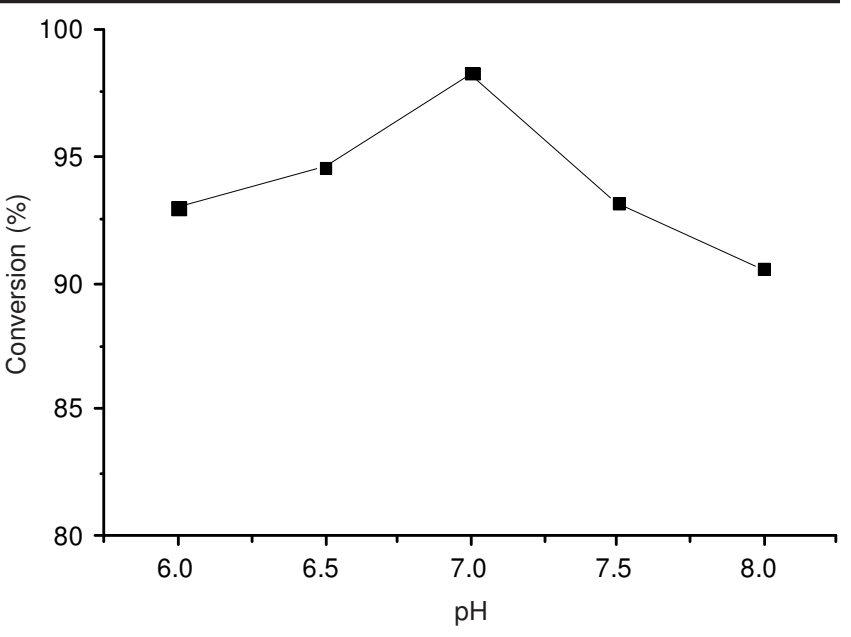

Fig. 4. Results of esterification under different buffer $\mathrm{pH}$

Effects of different solvent: Under the condition of $\mathrm{w}_{0}=$ 4 , molar ratio of acid to alcohol $1: 2$ at $40{ }^{\circ} \mathrm{C}$, we studied the effects of different solvent (isooctane, cyclohexane, hexane) on the esterification of hexanoic acid with ethanol. The results were listed in Fig. 5. Fig. 5 showed that the different solvent with DBSA can form the microemulsion and catalyze the esterification reaction. The conversion of hexanoic acid in isooctane was the highest, cyclohexane next and hexane was the last. This might be because of the reactants had different solubility in these solvent. The higher solubility was, the faster reaction rate was. So, choose a suitable solvent to improve the esterification reaction rate and equilibrium conversions are important.

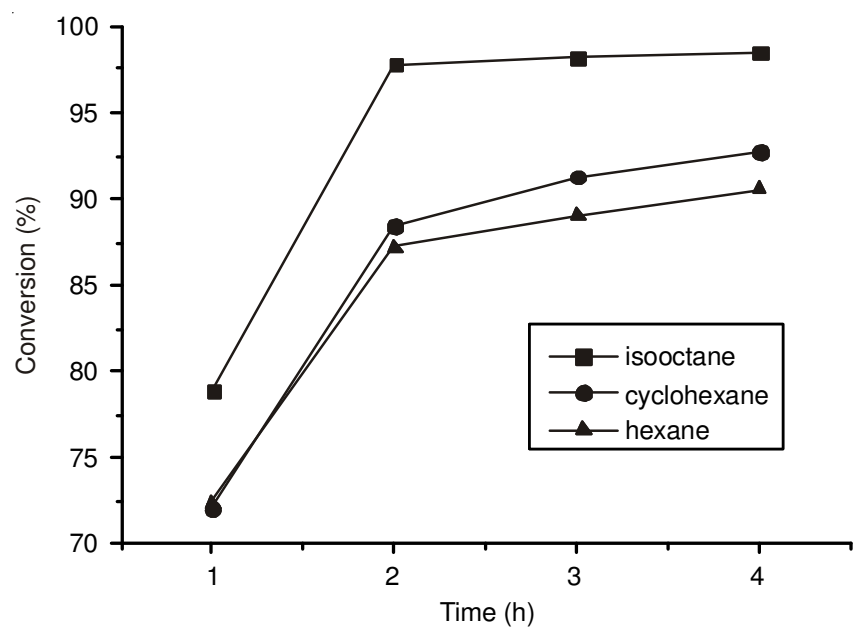

Fig. 5. Results of esterification under different solvent

Esterification of different acid and alcohol: The DBSA system was also applied to esterication with other alcohols and acids as substrate and the results were displayed in Fig. 6. As shown in Fig. 6, when a mixture of ethanol with butyric acid (isovaleric acid and hexanoic acid) and a mixture of hexanoic acid with ethanol (butanol and isoamyl alcohol) were subjected to esterification in DBSA microemulsion system at $313 \mathrm{~K}$ for $4 \mathrm{~h}$, these five equlibrium conversions were 98.2 , 94.1, 98.5, 92.5 and $96.0 \%$, respectively.

These results indicated that the conversion of substrates decreased at the equilibrium position with the alkyl chain and 


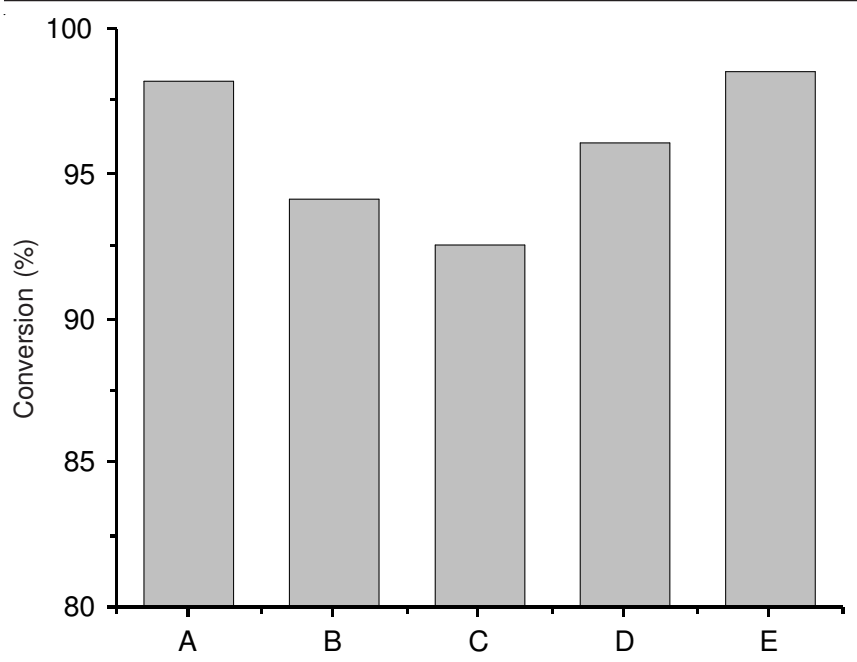

Fig. 6. Results of esterification under different acid with alcohol. $\mathrm{A}=$ ethyl butyrate; $\mathrm{B}=$ ethyl isovalerate; $\mathrm{C}=$ butyl hexanoate; $\mathrm{D}=$ isoamyl hexanoate; $\mathrm{E}=$ ethyl hexanoate

increased branch chain. This was because straight chain acid with alcohol, their space resistance was small, easy to collision and combine and conversion was high. But butyl hexanoate was also a straight chain ester, the situation was special and the specific reason was a subject of research. Based on the above results, we found that the DBSA reverse microemulsion system was preferential to esterification of the straight chain acid and short alkyl alcohol.

Effects of different reaction system: Subsequently, we studied the esterification in the different system and compared them with that in DBSA microemulsion system. The results were presented in Fig. 7. From Fig. 7, it is evident that the conversion in DBSA microemulsion system is much higher than that in other systems with the same substrates concentration after reaction proceeding $5 \mathrm{~h}$.

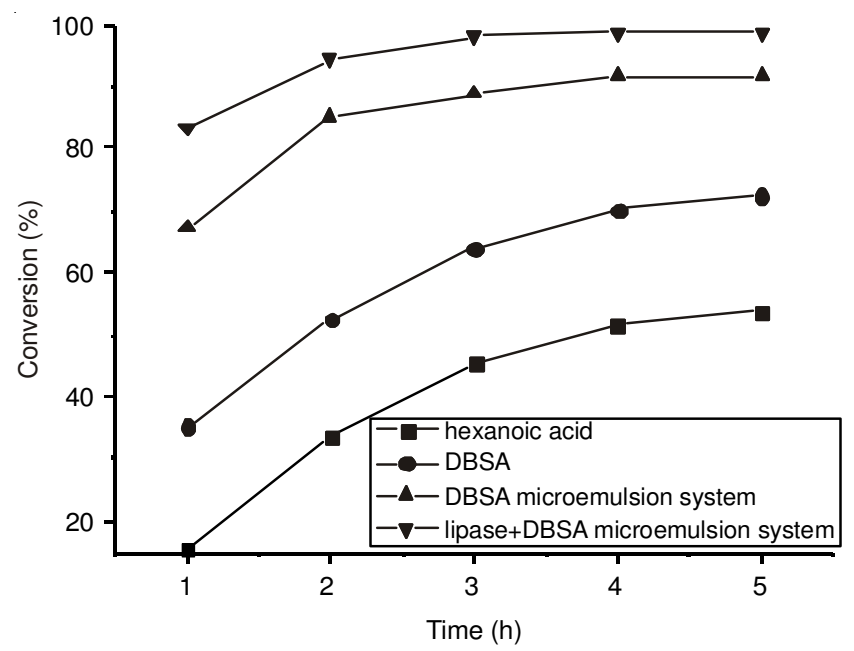

Fig. 7. Results of esterification under different reaction system

Fig. 7 showed that the hexanoic acid can catalyze esterification and equlibrium conversion was $53.9 \%$. This is because hexanoic acid is a kind of organic acid despite it cannot form microemulsion system. DBSA is a kind of Brønsted acid with the same acidity as $\mathrm{H}_{2} \mathrm{SO}_{4}$. The acidity of DBSA is more than that of hexanoic acid, so the catalysis effect of DBSA was better than hexanoic acid. DBSA, as a kind of surfactant, can also form thermodynamic stability microemulsion system, therefore, the combination of them not only possesses acid catalysis property, but also the collision opportunities of the reactant increase due to the large phase interface area. At the same time, the water produced by esterification reactions can be dissolved into the reverse micelle internal pool, which made the esterification reaction balance move to product direction and improved the esterification conversion. As a result, the equlibrium conversion in this system was higher $(91.7 \%, 5 \mathrm{~h})$ than that in separate system. From Fig. 7, it is also shown that the conversion of DBSA microemulsion system with lipase was higher than without lipase system. Lipase system could shorten the esterification reaction time of reaching the highest conversion. This result also indicated that DBSA microemulsion system with $\mathrm{w}_{0}=4$ could be helpful for lipase playing its activity and enhanced the reaction conversion.

Based on the above experimental results, it can be concluded that DBSA microemulsion system with lipase is the most effective system for the esterification conversion under mild conditions and have acid catalysis, microemulsion catalysis and enzyme catalysis triple mechanism.

\section{Conclusion}

We have described esterification reaction in DBSA/isooctane microemulsion system with lipase. This reaction system was found to be applicable to several esterification reactions under mild conditions. $91.7 \%$ yield of ethyl hexanoate was reached in DBSA microemulsion system without lipase after $5 \mathrm{~h}$ on the esterification of ethanol and hexanoic acid. Lipase in DBSA microemulsion system could improve the conversion of hexanoic acid $(98.1 \%)$ and shorten reaction time $(3 \mathrm{~h})$. The higher catalytic efficiency proved that DBSA microemulsion with lipase should be a promising reaction system for esterification reactions. The proton acid (DBSA) catalyzed, microemulsion catalyzed and enzyme catalyzed ternary mechanism was proposed in this microemulsion system.

\section{REFERENCES}

1. S.H. Krishna, A.P. Sattur and N.G. Karanth, Process Biochem., 37,9 (2001).

2. F. Ergan, M. Trani and G. Andre, J. Am. Oil Chem. Soc., 68, 412 (1991).

3. R. Perraud and F. Laboret, Appl. Microbiol. Biotechnol., 44, 321 (1995).

4. Y. Xu and K.C. Zhang, Chinese Wine, 4, 33 (1997) (in Chinese).

5. L.J. López Giraldo, M. Laguerre, J. Lecomte, M.-C. Figueroa-Espinoza, N. Barouh, B. Baréa and P. Villeneuve, Enzyme Microb. Technol., 41, 721 (2007).

6. H.F. Zhu, H.J. Zhang and N. Ma, Chem. Ind., 9, 23 (2008) (in Chinese).

7. J. Ni and F.C. Meunier, Appl. Catal., 333, 122 (2007).

8. F.M. Menger and A.R. Elrington, J. Am. Chem. Soc., 113, 9621 (1991).

9. F. Currie, K. Holmberg and G. Westman, Colloid. Surf. A, 182, 321 (2001).

10. K. Manabe, S. Iimura, X.-M. Sun and S. Kobayashi, J. Am. Chem. Soc., 124, 11971 (2002).

11. V.I. Mil'to, V.Yu. Orlov and G.S. Mironov, Kinet. Catal., 44, 747 (2003).

12. S. Iimura, K. Manabe and S. Kobayashi, Org. Lett., 5, 101 (2003).

13. D.X. Han, Y. Chu, L.K. Yang, Y. Liu and Z. Lv, Colloid. Surf. A, 259, 179 (2005).

14. M.J. Han, Y. Chu, D.X. Han and Y.J. Liu, J. Colloid Interf. Sci., 296, 110 (2006).

15. Y.H. Wang, Y. Chu, J.L. Liu, Z.X. Lü, Y.Y. Liu and Z.S. Wu, Chem. J. Chin. Univ., 25, 1684 (2004).

16. S.Y. Ma, Y. Chu and Z.X. Lv, Acta Chim. Sinica, 15, 1523 (2006).

17. Y.C. Han and Y. Chu, J. Mol. Catal. Chem., 237, 232 (2005).

18. Q.Y. Pan, Q. Lu and Z.X. Cheng, Chem. Res. Appl., 3, 284 (2001) (in Chinese). 